\title{
SUR LA POSSIBILITÉ DE TRANSFORMER DIRECTEMENT EN ÉNERGIE ÉLECTRIQUE UNE PARTIE DE L'ÉNERGIE D'UNE VEINE FLUIDE
}

par G. REMENIERAS

Adjoint au Chef du Service des Etudes et Recherches Hydrauliques d'Electricité de France

\section{(COMMUNICATION PRESENTEE AU COMITE TECHNIQUE DE LA SOCIETE HYDROTECHNIQUE DE FRANCE DANS SA SEANCE DU 24 NOVEMBRE 1947)}

\section{A. GÉNÉRALITÉS}

Dans tous les générateurs électriques industriels la f.e m. est engendrée par le déplocement de conducteurs solides, en cuivre généralement, dans un champ magnétique. Peut-on concevoir et utiliser pratiquement un générateur dans lequel la f.e.m. serait produite directement par l'écoulement d'un fluide conducteur (le mercure par exemple) dans un champ magnétique? Telle est la question que je m'étais posée il y a bien des années en constatant que dans les usines hydroélectriques, il n'était possible de transformer en électricité l'énergie hydraulique disponible au pied de la conduite forcée qu'après l'avoir transformée en énergie mécanique par l'intermédiaire d'une turbine. Combien il serait plus élégant de disposer d'un générateur en quelque sorte "statique dans lequel l'eau rendue suffisamment conductrice ou pratiquement entrainant un fluide relativement bon conducteur, abandonnerait toute sa puissance à un bobinage fixe en défilant dans un champ magnétique ad hoc.

La solution du problème n'intéresse d'ailleurs pas que les ingénieurs de la Houille Blanche; leurs collègues "thermiciens» sauraient certainement alimenter en mercure sous pression l'électrogénérateur «fluidocinétique» ci-dessus, en utilisant à l'exclusion de toute machine tournante une chaudière et un injecteur, tous deux à mercure; ils pourraient ainsi prétendre - à tort d'ailleurs - transformer directement la chaleur en électricité. Bien entendu, comme tous ses congénères de style classique notre néogénérateur électrique serait réversible et en se muant en moteur deviendrait une pompe en quelque sorte «statique» car elle ne comporterait pas d'organe tournant.

$$
\star^{\star} \star
$$

Un premier examen de la question m'avait vite convaincu à la fois de la possibilité "de principe » de réaliser un électrogénérateur à mouvement de fluide et de la limitation extrêmement étroite de ses applications pratiques.

Comme je le montrerai plus loin, la puissance massique des machines de ce genre que l'on peut concevoir à priori est faible et leurs inconvénients nombreux. J'avais conclu, en définitive, que leur principe pourrait être appliqué à la mesure des débits de fluide et peut-être à quelques petites installations très spéciales.

Or, ces dernières années, la presse technique s'est fait l'écho des recherches, couronnées de succès, poursuivies en vue d'utiliser la f.e.m. induite par un champ magnétique dans un liquide (eau, sang) circulant dans un tuyau de faible diamètre pour mesurer le débit de celui-ci ; ces travaux ont été effectués, à notre connaissance, aux Etats-Unis (Laboratoire de la Columbia University à New-York) par KOLIN (11) (1) en vue semble-t-il d'expériences de biologie médicale et en Suisse par B. THURLEMANN (7) (1) qui a présenté devant l'Université de Fribourg une thèse sur ce sujet "à la suite des essais du Prof. F. DESSAUER et de la mise au point d'une pompe à mercure électromagnétique basés sur l'application de la loi de BIOT-SÁVART».

Ces circonstances $\mathrm{m}^{\prime}$ ont conduit à reprendre mes idées initiales pour les préciser à la lumière

\footnotetext{
(1) Les chiffres entre parenthèses reportent à l'index bibliographique placé à la fin de la présente note.
} 
des résultats ci-dessus. L'objet de la présente note est d'exposer les grandes lignes de ce travail préliminaire qui pourrait servir d'introduction à une étude plus poussée du problème. Pour alléger l'exposé, je me bornerai ici à l'examen des questions de principe sans aborder le détail du fonctionnement théorique et de la technologie des dispositifs envisagés; ceux-ci appelleraient des développements parallèles ò ceux élaborés depuis longtemps déjà pour les machines électriques classiques homologues et ne présentent pas de grandes difficultés pour le spécialiste; par contre, il serait essentiel de procéder à des essais en vue de se rendre compte des singularités que la nature fluide du conducteur induit ne manquera pas d'apporter aux conceptions usuelles.

\section{B. - L'ÉLECTROGÉNÉRATEUR FLUIDO-CINÉTIQUE}

Bien que l'application la plus probable et la plus immédiate de l'action d'un champ magnétique sur un fluide en mouvement soit la mesure de la vitesse de ce dernier à partir de la f.e.m. induite, il parait intéressant de mettre en lumière le principe d'un électrogénérateur basé sur le même principe: l'organe caractéristique du débitmètre électrique sera toujours en effet un dispositif de ce genre convenablement simplifié et adapté au but particulier poursuivi.

\section{1}

\section{Possibilité de réaliser un électrogénérateur fluido-cinétique}

La possibilité de produire sur des conducteurs liquides la majorité des phénomènes électromagnétiques qui nous sont familiers dans le cas de conducteurs solides est démontrée par les expériences de KOLIN (11 et 9) THURLEMANN (7) DESSAUER que i'ai signalées plus haut et aussi par les observations effectuées journellement sur les fours électriques industriels. Le phénomène du «pincement» qui consiste dans la réduction de section - qui peut aller jusqu'ò la rupture du circuit - d'une veine de métal en fusion sous l'action du champ magnétique produit par le courant qui la traverse est bien connu dans les fours à induction à basse fréquence. Les effets de brassage du métal en fusion dûs aux forces électromagnétiques s'observent dans la plupart des fours électriques; dans les fours à haute fréquence à creuset cylindrique on a constaté que, dans certaines conditions, une charge d'aluminium fondu semble être repoussée par les parois du creuset et tend à prendre une forme d'équilibre voisine du tronc de cône. Le colcul des lignes équipotentielles et des lignes de courant dans ces systèmes complexes est évidemment difficile, mais peut être abordé dans les cas simples en utilisant les équations de MAXWELL. D'ailleurs, tout comme l'ingénieur, calculant la stabilité d'un ouvrage, délaisse le plus souvent les lois de l'élasticité pour les régles de la résistance des matériaux, nous pourrons dans bien des cas appliquer en première approximation les lois élémentaires de l'induction aux lieu et place des équations ci-dessus.

Le champ électrique induit dans une particule se déplaçant à la vitesse $V$ dans un champ magnétique $d^{\prime} i n t e n s i t e ́ ~ H$ sera proportionnel au produit vectoriel $[\mathrm{V}, \mathrm{H}]$

D'autre part, la résistance interne de la machine et par suite sa puissance maximum, toutes choses égales d'ailleurs, dépendra de la résistivité électrique du fluide utilisé. Examinons les valeurs de ces différents paramètres qu'il semble possible de réaliser dans le générateur à mouvement de fluide et comparons-les à celles admises dans les machines électriques usuelles.

\section{c) Vitesse de déplacement du fluide.}

Il est possible de faire circuler un liquide dans un entrefer magnétique à des vitesses du même ordre que celles réalisées à la périphérie des générateurs tournants classiques soit :

15 d̀ $20 \mathrm{~m} / \mathrm{s}$ pour les dynamos normales

60 à $80 \mathrm{~m} / \mathrm{s}$ pour les turbo-dynamos

20 à $40 \mathrm{~m} / \mathrm{s}$ pour les alternateurs normaux 120 à $140 \mathrm{~m} / \mathrm{s}$ pour les turbo-alternateurs

$43 \mathrm{~m} / \mathrm{s}$ pour la dynamo unipolaire POIRSON

Au point de vue énergétique, il faut noter que l'énergie correspondant ou $\frac{V^{2}}{2 g} d u$ fluide dans l'entrefer n'est pas utilisable dans le générateur et devra être récupérée à la sortie (sous forme potentielle par un diffuseur par exemple). Cette énergie est relativement importante; pour une vitesse de $20 \mathrm{~m} / \mathrm{s}$, elle atteint déjà $20 \mathrm{~m}$. 4 de hauteur de fluide.

Ainsi, sous la chute du Portillon de $1.400 \mathrm{~m}$. l'énergie maximum susceptible d'être utilisée 
par le générateur correspondrait à une chute de : $1.400-20=1.380 \mathrm{~m}$. environ pour

$$
\text { et } \quad 1.400-720=680 \mathrm{~m} \text {. environ pour }
$$$$
V=120 \mathrm{~m} / \mathrm{s}
$$

Si par un artifice convenable, la pression de $1.400 \mathrm{~m}$. d'eau est transférée à une veine de mercure, la charge $h$ correspondante est de 1.400

$$
13,6
$$

à mouvement de mercure ne pourrait utiliser que l'énergie correspondant à :

$$
\text { et } \begin{aligned}
103-20 & =83 \mathrm{~m} \text {. pour } \vee=20 \mathrm{~m} / \mathrm{s} \\
\text { ef } & =21 \mathrm{~m} \text {. pour } \vee=40 \mathrm{~m} / \mathrm{s}
\end{aligned}
$$

On voit que des considérations énergétiques et aussi de perte de charge et de résistance à l'érosion conduiront en pratique à limiter la vitesse du fluide dans l'entrefer; dans le cas où le système serait utilisé en moteur, la limitation de la vitesse ne serait plus motivée que par la grandeur des pertes de charge et le risque d'érosion et éventuellement de cavitation.

\section{b) Intensité du champ magnétique.}

Rien ne s'oppose à ce que I'on admette dans l'entrefer parcouru par le fluide une induction du même ordre que dans les machines électriques usuelles (soit 8 à 16.000 gauss): peut-être pourra-t-on aller au delà dans certains générateurs du type unipolaire qui ne comportent pas de pulsation magnétique.

Toutefois, on remarquera que la hauteur e de l'entrefer et la vitesse $V$ du fluide étant connées, le débit q par mètre de longueur de l'entrefer est déterminé. Si nous prenons e $=10 \mathrm{~mm}$. ce qui est plutôt un maximum pour les machines classiques, on aura: $q=0,01 \vee$ soit $0,4 \mathrm{~m}^{3} / \mathrm{s}$. pour $\mathrm{V}=40 \mathrm{~m} / \mathrm{s}$.

Pour obtenir une puissance massique acceptable il faudra donc que la pression à l'entrée du générateur soit assez élevée.

\section{c) Résistivité des liquides.}

Lo résitivité du cuivre industriel est de $1,6 \mathrm{microhms} / \mathrm{cm}$.

Le seul métal liquide à la température ordinaire est le mercure dont la résistivité est de 95 microhms $/ \mathrm{cm}$., soit 60 fois environ celle du $\mathrm{Cu}(1)$.

(1) Elle est donc de l'ordre de celle des nickels-chromes utilisés pour la construction des résistances chauffantes électriques.
Pour obtenir dans un conducteur de mercure la même perte par effet Joule que dans un conducteur de cuivre, on devra donc adopter une section dont les dimensions linéaires seront $\sqrt{60}=7,65$ fois plus grandes que pour ce dernier.

Des alliages de métaux alcalins fondant à basse température seraient sans doute supérieurs au mercure pour les applications que nous avons en vue. Ainsi un alliage de $78 \%$ de potassium et $22 \%$ de sodium fond à $-12^{\circ} \mathrm{C}$.; sa résistivité serait de l'ordre du cinquième de celle du mercure. Le sodium dont on a envisagé l'emploi sous tube acier étanche pour remplacer les barres de cuivre dans les installations électrométallurgiques pourrait aussi à l'état fondu fournir une solution acceptable car sa résistivité serait de 6 à 7 fois seulement celle du cuivre.

Voici quelques résistivités de métaux en fusion :

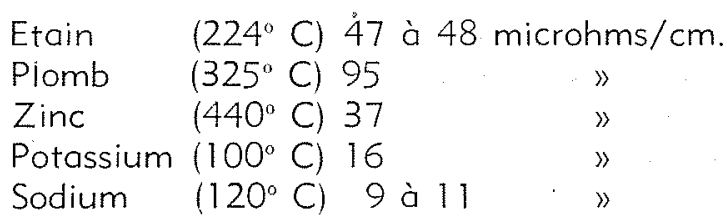

soit 6 à 7 fois celle du $\mathrm{Cu}$.

La résistivité des électrolytes est incomparablement plus forte que celle des métaux, aussi est-elle donnée le plus souvent en ohms par $\mathrm{cm}$.

\begin{tabular}{|c|c|c|}
\hline Acide sulfurique & 1,36 & ohms $/ \mathrm{cm}$ \\
\hline Potasse hydratée $(\mathrm{KOH})$ & 1,84 & $\gg$ \\
\hline Carbonate de $\mathrm{K}$ & 8,54 & $»$ \\
\hline Chlorure de $\mathrm{Na}$ & 4,66 & $»$ \\
\hline Sulfate de $\mathrm{Cu}$ & 29,37 & $»$ \\
\hline
\end{tabular}

Voici pour fixer les idées, les résistivités de quelques solutions aqueuses pour la concentrafion donnant le maximum de conductibilité :

La résistivité des électrolytes est donc au moins un million de fois plus forte que celle du cuivre; cela n'empêche pas de faire circuler dans les cuves d'électrolyse des courants de plusieurs milliers d'ampères, mais un électrogénérateur à mouvement d'électrolyte aura toujours une puissance massique très faible.

La résistivité des eaux naturelles est encore plus considérable et varie d'ailleurs beaucoup suivant la nature et la quantité de sels dissous qu'elles contiennent. La résistivité de l'eau distillée usuelle est de l'ordre de $10^{\circ} \mathrm{ohms} / \mathrm{cm}$. ; celle des eaux naturelles peut tomber vers $5.000 \mathrm{ohms} / \mathrm{cm}$. et au-dessous.

Ces fortes résisitivités ne font pas obstacle 
à l'utilisation du principe de l'électrogénérateur pour la mesure de la vitesse ou du débit des eaux naturelles, ainsi que l'ont montré les expériences de KOLIN et THURLEMANN. La f.e.m. induite est, toutes choses égales d'ailleurs, identique à celle qui se produirait dans le mercure par exemple, mais la résistance interne du générateur est telle que la puissance maximum qu'il est susceptible de développer est infime.

En conclusion, seul ie mercure et surtout certains métaux fondus sont susceptibles de fournir des générateurs de puissance massique notable; les électrolytes ne pourront guère être utilisés que dans des appareils de mesure.

\section{Polarisation des électrolytes.}

La polarisation est l'apparition d'une force contre-électromotrice due aux modifications produites tant à la surface dès électrodes qu'au sein de l'électrolyte (réactions secondaires, modifications des concentrations) par le phénomène d'électrolyse consécutif au passage du courant électrique. La polarisation interviendra dans tous les électrogénérateurs utilisant des électrolytes et notamment dans les débitmètres électriques traversés par de l'eau; en raison de la faiblesse des f.e.m. induites, ce phénomène ne peut être négligé, dans les appareils de mesure.

On le combattra, en courant continu, en employant des «électrodes impolarisables» (lesquelles sont constituées par une lame du métal du sel électrolysé) reliées électriquement au fluide en mouvement par une paroi poreuse (voir débitmètre THURLEMANN). II sera préférable, d'ailleurs, d'utiliser un courant alternatif bien symétrique à aussi haute fréquence et à aussi faible intensité que possible de façon à réduire au minimum la décomposition électrolytique. On choisira des électrodes ayant une «surtension électrolytique» faible (1) dans les conditions d'emploi et une grande surface; KOLIN fait usage dans un de ces débitmètres d'électrodes en platine platiné. La platinisation des électrodes est une opération bien connue des électrochimistes qui consiste à les recouvrir par voie

(1) Rappelons que l'on appelle surtension électrolytique l'excès du potentiel de décharge sur la valeur absolue de la f.e. $m$. de sens contraire qui serait $m$ 'se en évidence si, après électrolyse en quantité sensible, on faisait fonctionner le système comme élément de pile. C'est seulement lorsqu'il y a dégagement de gaz que se manifeste cette surtension; sa valeur dépend de la nature et de l'état physique de l'électrode, de la densité de courant, de la nature de l'électrolyte, etc... Voici, pour fixer les idées, quelques valeurs de la surtension d'H: Platine platiné .... 0.00 volts $\mathrm{Zn}: 0.70$ volts Platine poli ...... 0.09 volts $\mathrm{Hg}: 0.78$ volts électrolytique d'une mince couche de noir de platine; l'état de surface des électrodes est ainsi profondément modifié (leur surface efficace est égale à plusieurs centaines de fois leur surface réelle) et on obtient des surtensions électrolytiques très faibles (voir note 1).

Remarque. - Si l'on est amené à capter au moyen d'électrodes fixes des courants engendrés dans un liquide en mouvement, on observera une perte d'énergie correspondant à la «chute de tension aux balais» des machines classiques. Cette chute de tension est très faible.

A l'occasion de la mise au point de sa dynamo unipolaire, $M$. POIRSON (10) a mesuré une chute de tension de 0,12 volts seulement entre jet de mercure et cuivre pour une densité de courant dans le jet de $20.000 \mathrm{Amp} / \mathrm{cm}^{2}$; un balai cuivre-graphite donnait 0,3 volts pour une densité de $30 \mathrm{~A} / \mathrm{cm}^{2}$.

II

\section{Constitutrion schémafique d'un électrogénérateur Huido-cinétique}

Dans la recherche du schéma des divers types d'électrogénérateur possibles, il ne faut pas perdre de vue les considérations suivantes:

Dans ces appareils, l'induit sera constitué non par des faisceaux de conducteurs séparés et isolés montés en série ou en dérivation comme dans les machines électriques usuelles, mais par le liquide en mouvement qui pourra être assimilé en première approximation à un cylindre creux ou à une plaque se déplaçant en bloc à la vitesse moyenne du fluide. Il semble donc logique de chercher à réaliser des appareils homologues aux machines classiques pouvant être exécutés avec des induits conducteurs massifs en forme de disque ou de cylindre creux ou pleins. A notre connaissance il existe deux genres de machines répondant à cette condition :

$\left.1^{\circ}\right)$ les dynamos unipolaires dites aussi acycliques dont la dynamo POIRSON 150.000 A-14 V) installée au Palais de la Découverte à I'Exposition de Paris en 1937 (10) est une des réalisations les plus récentes.

$2^{\circ}$ ) les moteurs asynchrones à induit en courtcircuit. En fait, ces induits sont généralement exécutés en cage d'écureuil, mais la théorie et l'expérience montrent que de tels moteurs peuvent encore fonctionner avec un induit massif; ils sont employés comme équipage mobile dans certains wattmètres à champ tournant 
(Hartmann et Braun), et on a réalisé des moteurs asynchrones à induit en acier massif pour le démarrage des commutatrices de forte puissance.

D'autre part, dans les machines électriques classiques, les conducteurs induits sont animés d'un mouvement de rotation autour d'un axe. Dans les électrogénérateurs a mouvement de fluide, il sera plus pratique d'imprimer au conducteur élémentaire une translation amenant le liquide de l'entrée à la sortie de la machine. On pourrait notamment passer de la machine classique au générateur fluidocinétique en développant (1) sur un plan la circonférence de I'induit et de l'inducteur de la première. Au point de vue constructif et aussi pour obtenir une symétrie aussi parfaite que possible des champs et des courants, il semble plus avantageux de concevoir l'électrogénérateur fluidocinétique de la façon suivante.

Le fluide circulerait longitudinalement dans l'entrefer compris entre deux cylindres concentriques en métal magnétique dans lequel des bobinages ad hoc maintiendraient un champ radial continu ou alternatif, unipolaire ou multipolaire (fig. 4 et 5). L'induit serait donc constitué par un cylindre creux de fluide animé d'un mouvement de translation parallèlement à l'axe dudit cylindre comme nous le montrerons plus loin. On peut considérer que ce mode de construction dérive de la machine obtenue par développement plan des générateurs classiques en courbant suivant un demi-cercle les génératrices de l'entrefer plan de ces machines et en juxtaposant de part et d'autre d'un diamètre commun deux systèmes identiques (voir figures 2 et 2 bis).

111

\section{Electrogénérateur fluidocinétique type $A$ dérivé de la dynamo unipolaire}

\section{a) Principe.}

Essayons, par exemple, de réaliser un système homologue à la dynamo POIRSON (10) dont le schéma est brièvement rappelé ci-après : L'induit est un cylindre en acier massif qui tourne dans un champ magnétique unipolaire créé par 2

\footnotetext{
(1) C'est ainsi qu'ont été conçues certaines pistes de lancement électrique des avions aux Ełats-Unis. La piste de 414 mètres de long de PATUXTENT RIVER peut être cons.deree comme le développement sur un plan de la périphérie d'un induit à cage d'écureuil de 132 mètres de diamètre de moteur asynchrone; le chariot de lancement de l'avion a $3 \mathrm{~m}$. 50 de long et $1 \mathrm{~m}$. 05 de large et constique le developpement plan dune partie de linducteur du moteur ci-dessus. A vide, le chariot atteint une vitesse de plus de $400 \mathrm{~km} . / \mathrm{h}$. au bout d'un parcours de 150 mètres.
}

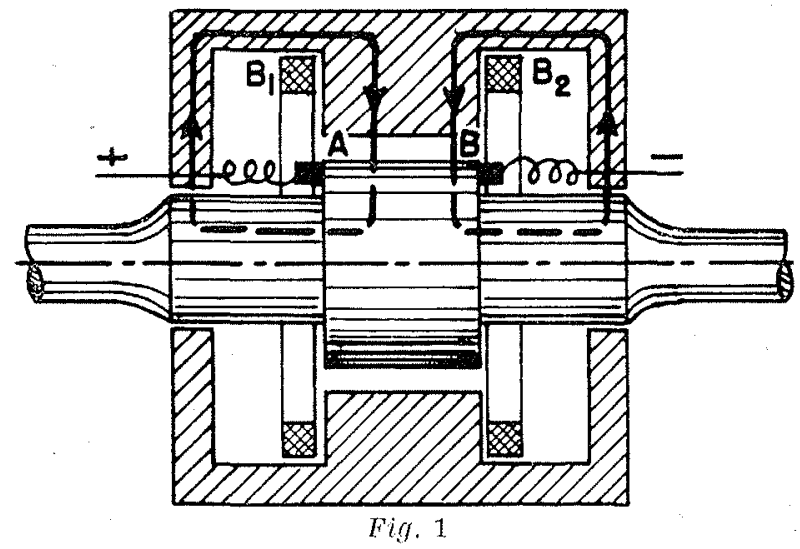

Dinamo unipolaire POIRSON

bobines $B_{1}$ et $B_{2}$ enroulées en forme de couronnes concentriques à l'induit. Il est facile de montrer que la f.e.m. continue créée entre les deux extrémités d'une génératrice telle que $A B$ de l'induit a pour expression:

$$
\begin{aligned}
& E=B \mid V 10-8 \\
& E: \text { f.e.m. en volts } \\
& B: \text { induction en gauss } \\
& I: \text { longueur de la génératrice } A \text { B en } \mathrm{cm} \text {. } \\
& V: \text { vitesse périphérique en } \mathrm{cm} . / \mathrm{s} \text {. }
\end{aligned}
$$

On peut aussi employer la formule équivalente:

$$
E=N \Phi 10-8
$$

dans laquelle $N$ est le nombre de tours par seconde de l'induit et $\$$ le flux total traversant sa surface périphérique.

Le courant continu produit est recueilli par

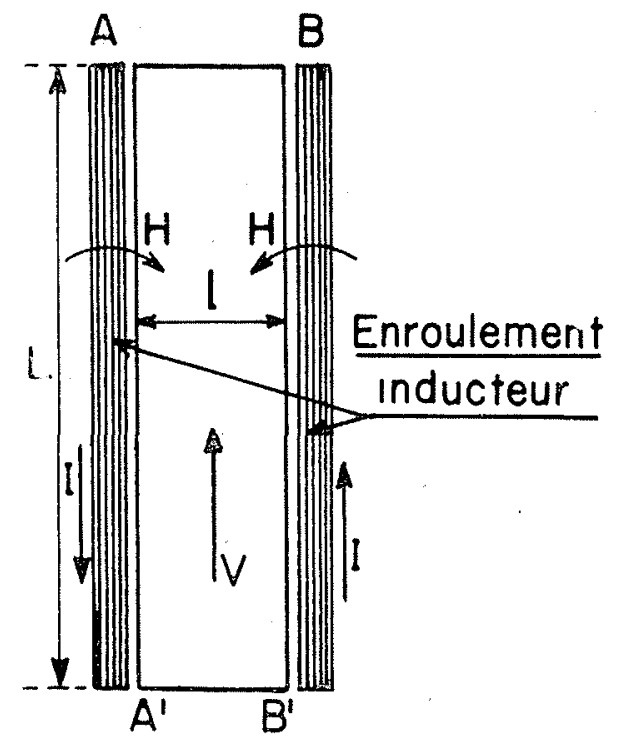

Fig. 2

Développement plan

de l'induit et de l'inducteur de la dynamo POIRSON 
des couronnes de balais placées en A et B sur les faces latérales de l'induit.

Pour passer de cette machine à l'électrogénérateur fluidocinétique équivalent faisons les transformations géométriques envisagées dans le paragraphe précédent :

En développant l'entrefer suivant un plan on obtient le système de la figure 2 qui pourrait constituer l'électrogénérateur cherché. Le fluide. circulerait avec la vitesse moyenne $V$ dans un

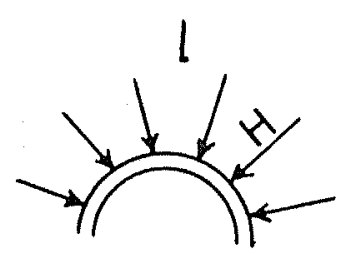

Fig. 2 bis

Cintrage en demi-cercle de l'entrefer de la fig. 2

entrefer plon isolé électriquement d'épaisseur e et de largeur I dans lequel il serait facile d'établir un champ magnétique perpendiculaire à $V$.

On recueillerait entre les 2 bandes conductrices latérales fixes $A A^{\prime}$ et $B B^{\prime}$ une f.e.m. :

$$
E=\int_{0}^{l} B V d l \text { (B pouvant être une }
$$

fonction de 1)

Si l'on pose $\mathrm{AA}^{\prime}=\mathrm{L}$ et si $\mathrm{t}$ désigne le temps mis par l'élément de fluide $A^{\prime} B^{\prime}$ pour venir en $A B$ on $a$ :

$$
E=\frac{1}{t} \int_{0}^{l} \mathrm{BL} d \mathrm{l}
$$

L'intégrale représente évidemment le flux qui traverse l'entrefer de sorte que l'on a :

$$
E=-\frac{\Phi}{i}
$$

Mais il est facile de voir que les bornes $A A^{\prime}$ et $B B^{\prime} d^{\prime} u n$ tel générateur sont mises en courtcircuit par la partie du fluide qui se trouve en dehors du champ magnétique; la résistance de ce court-circuit pourrait être évaluée en traçant théoriquement ou expérimentalement le faisceau des lignes équipotentielles et des lignes de courant dans les régions $A B$ et $A^{\prime} B^{\prime}$; il semble possible d'augmenter cette résistance par divers artifices tel que la division de la veine liquide dans ces régions par des cloisons isolantes parallèles à $A A^{\prime}$.

Quoiqu'il en soit le système considéré comme générateur d'électricité paraît condamné à un médiocre rendement que seuls quelques essais permettraient d'estimer. II peut néanmoins présenter quelque intérêt comme élément débitmétrique ou encore pour transformer en chaleur de l'énergie disponible sous forme hydraulique. Supposons, en effet, que par un artifice quelconque l'énergie hydraulique disponible au pied d'une conduite forcée soit utilisée à mettre en circulation dans l'électrogénérateur ci-dessus mis en court-circuit en réunissant (1) $A A^{\prime}$ et $B B^{\prime}$ un volume limité de mercure. Toute l'énergie hydraulique serait ainsi transformée en chaleur par effet Joule dans le mercure avec un rendement probablement excellent. La température d'ébulition du mercure à la pression atmosphérique étant de $357^{\circ}$ on voit qu'il serait relativement aisé d'établir des échangeurs de température pour utiliser la chaleur ainsi engendrée.

On remarquera que nous n'avons fait aucune hypothèse sur la nature du champ magnétique établi dans l'entrefer. Il est bien évident que le système peut fonctionner en courant alternatif comme en courant continu, sous réserve de tenir compte des effets de la self induction. Dans ce cas on pourrait obvier en partie à l'inconvénient de ce genre d'appareil de ne donner que de faibles f.e.m. mais des courants intenses en lui associant un transformateur élévateur.

L'électrogénérateur est reversible, c'est-à-dire que si l'on établit une différence de potentiel entre les électrodes $A A^{\prime}$ et $B B^{\prime}$ le liquide sera mis en mouvement conformément aux lois de l'électromagnétisme et nous aurons réalisé une pompe sans organe tournant; son rendement sera sans doute faible en raison notamment du court circuit qui s'établit dans les régions $A A^{\prime}$ et $\mathrm{BB}^{\prime}$, mais peut-être une pompe à mercure réclisée sur ce principe trouverait-elle quelques applications dans les appareils de physique.

On peut remarquer que l'électrogénérateur mis en court-circuit et introduit dans une canalisation équivaut à une perte de charge proportionnelle au carré de la vitesse du fluide et on pourrait envisager son utilisation comme élément amortisseur ou destructeur d'énergie dans des fluides suffisamment conducteurs.

Théoriquement du moins, un électrogénérateur type A excité en courant continu pourrait être utilisé pour électrolyser à l'intérieur même de

(1) Par exemple en couplant convenablement deux générateurs identiques. 
I'appareil convenablement adapté, un électrolyte utilisé comme fluide moteur et circulant en circuit fermé. Cette mise en circulation pourrait se faire par exemple par l'énergie hydraulique disponible au pied d'une conduite forcée de sorte que l'appareil transformerait celle-ci en énergie chimique sans l'intermédiaire d'aucune machine électrique ou hydraulique. Bien entendu, la f.e.m. de l'électrogénérateur devrait être supérieure à la tension de décomposition électrolytique de l'électrolyte employé, mais ce ne serait pas une limitation du système en pratique car celle-ci ne dépasse jamais quelques volts (1) $(1,67$ volts pour l'eau). Dans un tel cas on pourrait semble-t-il augmenter la résistance de la mise en court-circuit du générateur dans les régions $A A^{\prime}$ et $B B^{\prime}$ par l'artifice suivant qui mérite d'être signalé.

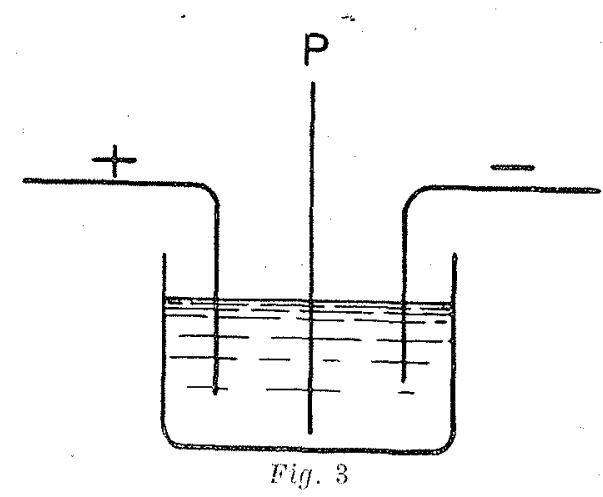

Considérons un voltamètre polarisable (fig. 3). Plaçons entre les électrodes une lame conductrice $P$ (en platine par exemple) soit $U$ la tension appliquée aux bornes du voltamètre et e la tension de décomposition électrolytique. Si $U>2$ e, la lame $P$ fonctionne comme électrode bipolaire c'est-à-dire que l'une de ses faces joue le rôle de cathode et l'autre d'anode et l'on se trouve en présence de 2 voltamètres en série. Mais si $U<2$ e la lame $P$ se comporte comme une lame isolante. Le système pourrait probablement être utilisé aussi avec un appareil excité en courant alternatif.

\section{b) Mode de réalisation pratique d'un électro- générateur type $A$.}

Le type d'électrogénérateur obtenu par développement plan de la machine classique homo-

(1) Elle est même théoriquement nulle forsqu'on emploie comme électrodes le métal même du sel électrolysé (électrodes non polarisables); ainsi, dans le raffinage du cuivre, la tension aux bornes d'un élément est en pratique de 0,13 volts pour la densité de courant utilisée de 1 à 3 ampères par dm². logue qui nous a servi à mettre en lumière les principales caractéristiques du système ne serait pas généralement d'une construction très commode. La machine représentée par les figures 4 et 5 que j'ai imaginée, serait sans doute plus pratique; elle dérive de la première par appli-

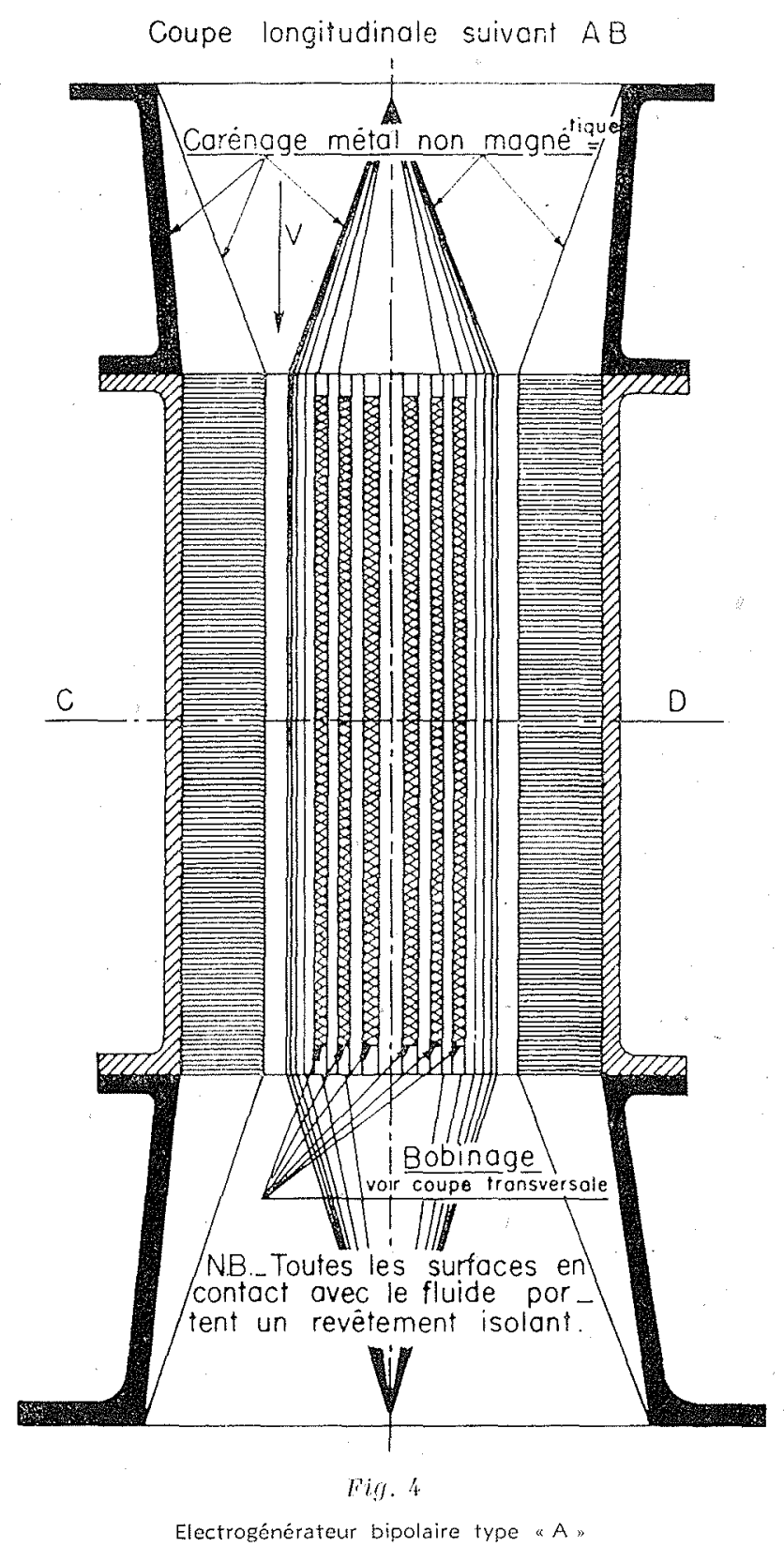

cation de la deuxième transformation géométrique proposée dans le paragraphe précédent.

L'inducteur est un cylindre en acier massif ou feuilleté suivant qu'il est alimenté en courant continu ou alternatif; il porte un bobinage analogue à celui des turbo-alternateurs et crée 
un champ radial comme dans ces dernières machines: Ce champ magnétique se ferme par une culasse constituée par une couronne concentrique à l'inducteur et de même métal, mais ne comportant aucun bobinage. Dans l'entrefer compris entre les 2 cylindres revêtus d'un isolant électrique circule parallèlement à leur axe, donc perpendiculairement au champ magnétique, le fluide moteur.

11 est clair que les courants induits dans les 2 demi-couronnes de diamètre $X X^{\prime}$ sont en opposition ; on disposera donc en $X$ et $X^{\prime}$ deuX

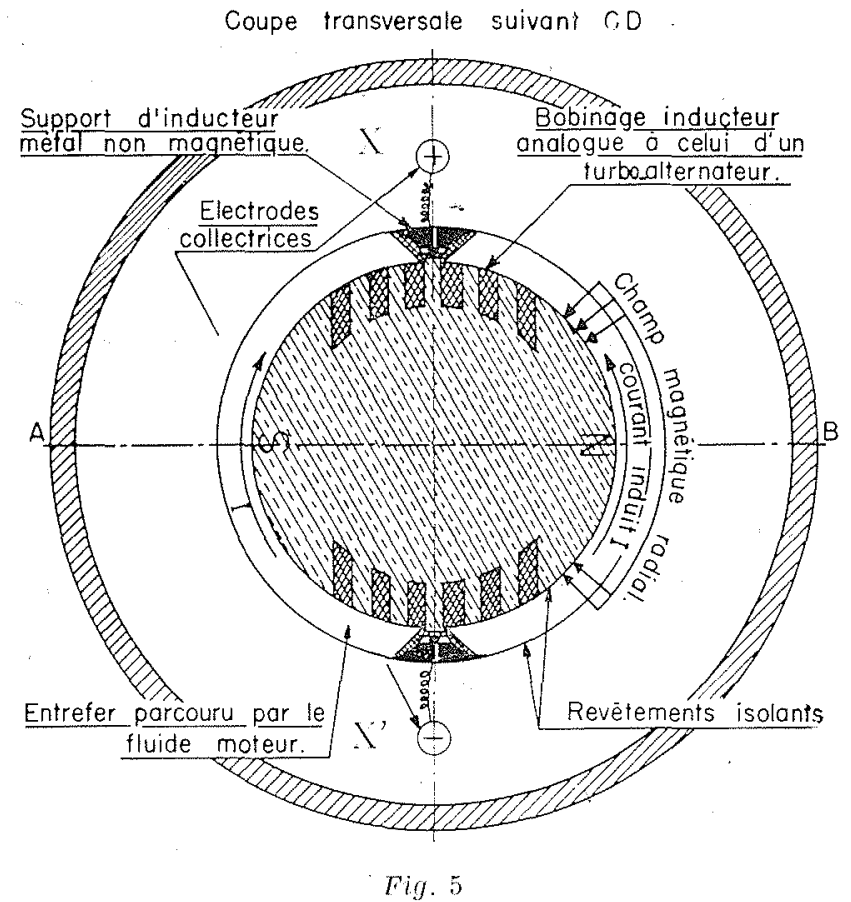

Elecirogénérateur bipolaire fype \& $\mathrm{A}$.

électrodes collectrices longitudinales qui seront les bornes de la machine.

Il serait facile de réaliser des machines multipolaires suivant le même schéma; la machine à 2 p pôles aurait $p$ lignes d'électrodes collectrices telles que $X X^{\prime}$ qui seraient reliées en parallèle.

Le raccordement de la machine aux canalisations d'amenée et de sortie du fluide se fait sans difficulté comme le montre la fig. 4 par l'intermédiaire de carénages destinés à diminuer les pertes de charge. Au droit de ces carénages, on pourra disposer s'il y a lieu une série de cloisons radiales destinées à augmenter la résistance du court-circuit formé par le fluide qui entoure les extrémités de l'inducteur.
On pourra combattre la réaction d'induit très importante dans ce genre de machine par un système analogue à celui employé sur la dynamo POIRSON.

\section{c) Ordre de grandeur des tensions et puissances réalisables :}

Pour fixer les idées, admettons :

$1,00 \mathrm{~m}$. pour le diamètre moyen de l'entrefer. $10 \mathrm{~mm}$. comme épaisseur de l'entrefer.

$20 \mathrm{~m} . / \mathrm{s}$. comme vitesse du mercure que nous adoptons comme fluide moteur.

13.000 gauss comme induction moyenne dans l'entrefer (en courant continu il n'y a pas de pertes par hystérésis et courants de FOUCAULT dans le fer de ces machines).

La f.e.m. dans chacune des demi-circonférences (de $157 \mathrm{~cm}$. de longueur) en opposition qui forment le conducteur élémentaire sera: $\mathrm{E}=\mathrm{B} \mid \mathrm{V} \times 10-8=13.000 \times 157 \times$ $2.000 \times 10-8=41,3$ volts

La f.e.m. de la machine est donc de 41,3 volts.

Résistance ohmique de l'induit en mercure pour une longueur de fer de 1 mètre:

$$
\begin{aligned}
\mathrm{s}=100 \times 1 & =100 \mathrm{~cm}^{2}=10 \mathrm{~mm}^{2} \\
1 & =157 \mathrm{~cm} .
\end{aligned}
$$

Pour le mercure: $=96$ microhms $/ \mathrm{cm}$.
où $R=96 \frac{157}{100}=156 \cdot 10-6 \mathrm{ohm}$ pour chaque demi-induit soit pour les 2 en parallèle: $78 \times 10-6 \mathrm{ohm}$.

Cette valeur de la résistance est grossièrement approchée par excès puisqu'elle ne tient pas compte des courts-circuits créés par le fluide aux deux extrémités de la machine. Si l'on réunit par une résistance négligeable les 2 bornes $X$ et $X^{\prime}$ (marche en chaudière électrique) le courant de court-circuit dans le circuit extérieur de résistance nulle ainsi constitué sera (en négligeant la réaction d'induit) $\frac{41,3}{78 \times 10^{-6}}=$ 530.000 Amp., soit une densité de courant de 53 Amp. par $\mathrm{cm}^{2}$, ce qui correspond c̀ une puissance :

$$
\mathrm{W}=\mathrm{El}=530.10^{3} \times 41,3=21.900 \mathrm{KW}
$$

Dans la marche en générateur la puissance maximum dans le circuit extérieur sera obtenue lorsque celui-ci aura une résistance égale à la 
résistance interne de la machine; elle correspondra donc à une intensité de 265.000 ampères et à une puissance

$$
265 \times 10-3 \times \frac{41,3}{2}=5.475 \mathrm{kw}
$$

Si l'on définit la pleine charge comme le régime où les pertes internes par effet Joule sont égales à $10 \%$ de la puissance fournie, celle-ci sera de :

$$
\begin{gathered}
W=U 1=41,3 \times 0,9\left(\frac{4,13}{2} \times 10+6\right)= \\
37,17 \times 53.000=1.970 \mathrm{kw}
\end{gathered}
$$

Le remplacement du mercure par du sodium fondu conduirait à des puissances 6 fois plus fortes.

La réaction d'induit et l'influence des courtscircuits internes de la machine réduiraient probablement tous ces chiffres dans une proportion que seule l'expérience directe pourrait préciser.

Supposons que nous remplaçions le mercure comme fluide moteur par une solution aqueuse de potasse caustique qui au titre de $29,4 \%$ présente une résistance particulièrement favorable (1) de $1,84 \mathrm{ohm} / \mathrm{cm}$. La résistance interne de la machine sera multipliée par:

$$
\frac{1,84 \times 10^{\circ}}{96}=19.160
$$

Les courants et les puissances calculés pour le générateur à mercure seront divisés par ce même facteur et deviendront :

$$
\begin{aligned}
& \text { Courant de court-circuit: } 27,7 \mathrm{Amp} \text {. } \\
& \begin{array}{l}
\text { Puissance correspondante: } \\
\text { Puissance maximum aux }
\end{array} \\
& \text { bornes ........... } 0,28 \mathrm{kw} \\
& \text { Puissance correspondante } \\
& \text { à } 10 \% \text { de perte Joule: } 0,1 \mathrm{kw}
\end{aligned}
$$

On voit que l'emploi des électrolytes conduit à des puissances aux bornes infimes (bien inférieures à la dépense d'excitation qui serait de l'ordre de 3 à $5 \mathrm{kw}$ pour la machine envisagée) et ne peut être retenu pour un électrogénérateur. Par contre, convenablement simplifiée cette dernière machine constituerait un élément débitmétrique assez sensible; pour une vitesse de $2 \mathrm{~m}$./s. et une induction ramenée à 1.300 gauss,

\footnotetext{
(1) Une solution de $\mathrm{SO}^{+} \mathrm{H} 2$ donnerait : $P=0.845 \mathrm{ohm} / \mathrm{cm}$. à la concentration de $15 \%$
} mais ces solutions seraient très corrosives. la f.e.m. serait encore de 0,4 volts, ce qui permettrait (après étalonnage) de bonnes mesures avec un simple millivoltmètre de forte résistance.

Pression à l'entrée de l'électrogénérateur à mercure pour la puissance $W=1.970 \mathrm{kw}$. Si $\because$ est le rendement de la machine, $Q$ son débit en tonnes par seconde et $\mathrm{H}$ la chute de pression en mètres de mercure dans la machine la puissance électrique aux bornes a pour expression:

$$
\mathrm{W}=r_{\mathrm{QH}} \times 9,81
$$

$\%_{i}$ étant le rendement du générateur électrique d'où:

$$
H=\frac{W}{9,81 \% Q}
$$

Or, le débit $Q$ de l'électrogénérateur considéré pour une vitesse de mercure de $20 \mathrm{~m}$./s. est de $0,63 \mathrm{~m}^{3} / \mathrm{s}$., soit $8,5 \mathrm{t} / \mathrm{s}$. d'où :

$$
H=\frac{1.970}{9,81 r_{i} \times 8,5}=\frac{23,6}{\pi_{i}} \text { mètres de } \mathrm{Hg}
$$$$
\text { soit } \frac{32}{n} \mathrm{~kg} / \mathrm{cm}^{2} \text { ou } \frac{320}{n} \mathrm{~m} \text {. d'eau. }
$$$$
11 \text { est bon de noter que le } \frac{V}{2 \mathrm{~g}} \text { à la sortie de }
$$

la machine représente une hauteur de 20 mètres de mercure à peine inférieure à la contrepression d'origine électromagnétique. Il est donc indispensable de récupérer cette énergie et au point de vue du rendement global, il serait sans doute avantageux de réduire la vitesse du fluide dans l'entrefer, car la perte de charge résultant du frottement du liquide sur les parois de ce dernier est importante.

\section{IV}

\section{Electrogénérateur fluidocinétique type $B$ dérivé du moteur asynchrone à champ tournont à induit en cage d'écureuil}

\section{a) Moteur asynchrone.}

Essayons, par exemple, de réaliser un système homologue à un moteur asynchrone triphasé à induit en cage d'écureuil ou plus exactement à enroulement induit constitué par un cylindre creux en conducteur massif. Suivant la méthode utilisée dans le paragraphe précédent, nous pouvons tout d'abord développer sur un plan la surface de l'induit et de l'inducteur. On obtient 


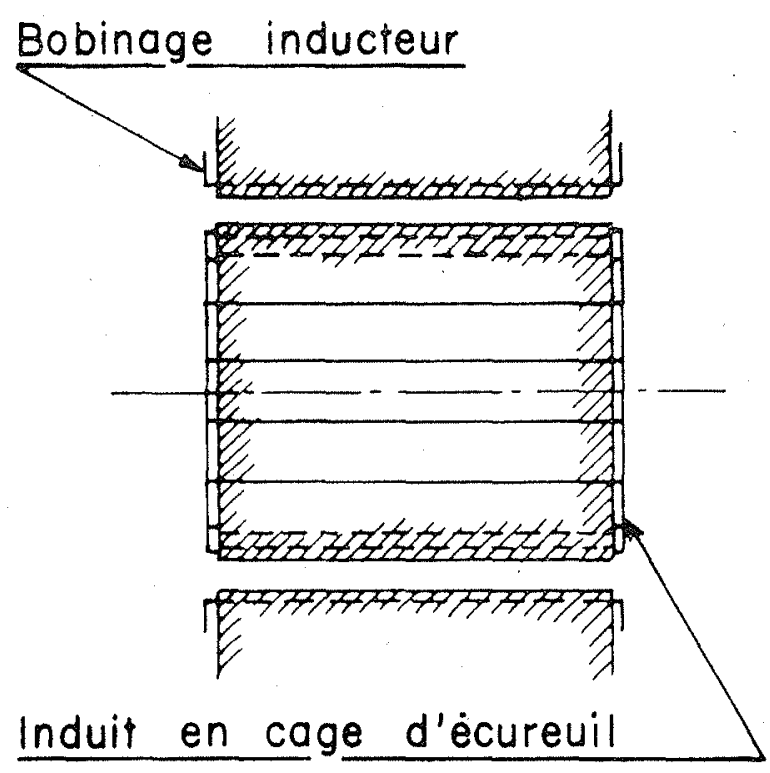

Fig. 6

ainsi le système figure 7 utilisé pour les pistes de lancement des avions que nous avons signalé à la page 7 ; le champ tournant est remplacé par un «champ glissant » à une vitesse égale en $\mathrm{m}$./s. à la longueur du pas polaire du bobinage en mètres multiplié par la fréquence du courant d'alimentation. Si nous remplissons l'entrefer avec un liquide conducteur - du mercure par exemple - celui-ci jouera, plus ou moins parfaitement, le rôle des barres de la cage d'écureuil d'un moteur classique et si l'enroulement inducteur est alimenté, en courant triphasé par exemple, le mercure sera entraîné parallèlement aux arêtes $\mathrm{AA}^{\prime}$ et $\mathrm{BB}^{\prime}$ ò une vitesse égale à la vitesse du champ glissant diminué d'un certain glissement qui sera fonction de la pression que devra vaincre le liquide ( $c^{\prime}$ est ici I'homologue du couple du moteur classique) et de la résistance électrique de la veine fluide.

A la vérité, l'électrogénérateur à fluide ainsi réalisé diffère du moteur à induit massif par le fait que, à l'inverse de ce qui se passe dans ce dernier, les lignes de courant induit peuvent se fermer en dehors du champ à travers le liquide arrivant et sortant de la machine. On peut tenter de réduire cette perturbation par les ortifices déjà signalés à propos de l'électrogénérateur type $A$ et seule l'expérience directe permettra d'apprécier son importance.

L'électromoteur ainsi réalisé constitue une pompe puisque comme dans les pompes centrifuges, il sera toujours possible de transformer en énergie potentielle par l'intermédiaire d'un divergent bien proportionné l'énergie cinétique du fluide mis en vitesse dans l'entrefer. Comme dans un moteur asynchrone à induit massif, le couple au démarrage est faible; il est vraisemblable que ces pompes ne pourront s'amorcer qu'avec une contrepression au refoulement inférieure au pouvoir manométrique de la machine à pleine charge.

D'après ce que nous avons vu pour l'électrogénérateur type $A$ le système ne pourrait guère fonctionner qu'avec du mercure ou certains métaux fondus (application à la coulée sous pression?) ; si son rendement se révèlait acceptable, peut-être pourrait-il trouver quelques applications pratiques en raison de sa simplicité et de sa robustesse.

\section{b) Génératrice asynchrone.}

On sait que si un moteur asynchrone à champ tournant est entraîné mécaniquement à une vitesse supérieure à celle du synchronisme, il se transforme en génératrice asynchrone restituant ou réseau électrique (au rendement près) l'énergie qui lui est fournie sur son arbre. Le rendement est excellent avec des induits bobinés; nous manquons de renseignements sur sa valeur dans le cas d'induits à cage d'écureuil et d'induits massifs

Il n'est pas douteux pourtant que la marche en génératrice est encore possible; le système est en effet assimilable à un transformateur dont le côté alimentation n'aurait qu'une seule spire. Dès lors, supposons que l'énergie poten-

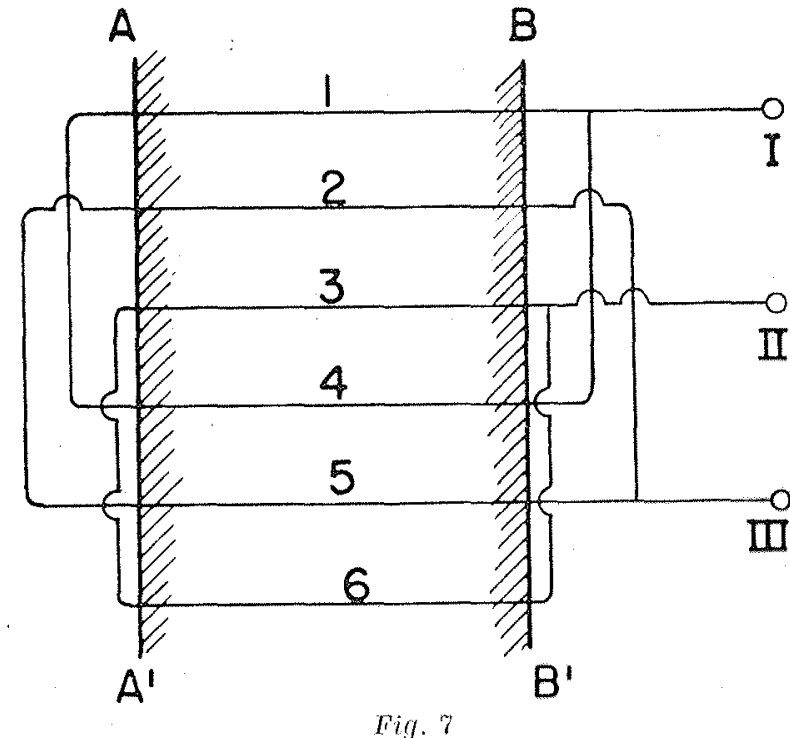


tielle disponible au bas d'une conduite forcée ou à la sortie d'une chaudière soit transférée par un artifice quelconque à une veine de mercure circulant à grande vitesse dans l'entrefer d'un électromoteur type B à une vitesse supérieure à la vitesse de synchronisme du champ glissant produit par l'inducteur de ce dernier; nous aurons réalisé une génératrice asynchrone en quelque sorte «statique» permettant de transtormer en énergie électrique l'énergie hydraulique contenue dans le fluide sans passer par l'intermédiaire d'une machine tournante; ici encore certaines applications pratiques pourraient être envisagées si à l'expérience le rendement se révélait acceptable.

\section{c) Mode de réalisation pratique d'un électro- générateur type $B$.}

Il semble avantageux d'adopter une construction telle que celle représentée par la figure 9 analogue à celle proposée pour le type A. Les figures 6 à 9 montrent qu'on passe de la machine classique de la figure 7 à celle de la figure 9 :

$\left.1^{\circ}\right)$ en courbant en demi-cercle la fibre neutre de la section transversale de l'inducteur et de I'induit préalablement développés, de façon que les faces $A C$ et $D B$ se trouvent sur un même diamètre CABD (fig. 8).

$2^{\circ}$ ) en juxtaposant de part et d'autre de ce diamètre commun deux systèmes identiques dont les bobinages inducteurs pourront être couplés à volonté de façon à engendrer dans chaque demispire de l'induit, soit des fie.m. en série, soit en opposition (les courants se fermeraient alors par le plan diamétral $\mathrm{CABD}$ qui est I'homologue des cercles d'extrémité de la cage d'écureuil).

II semble que la première solution serait préférable, car elle permettrait de constituer le bobinage par des séries de spires concentriques

(b)

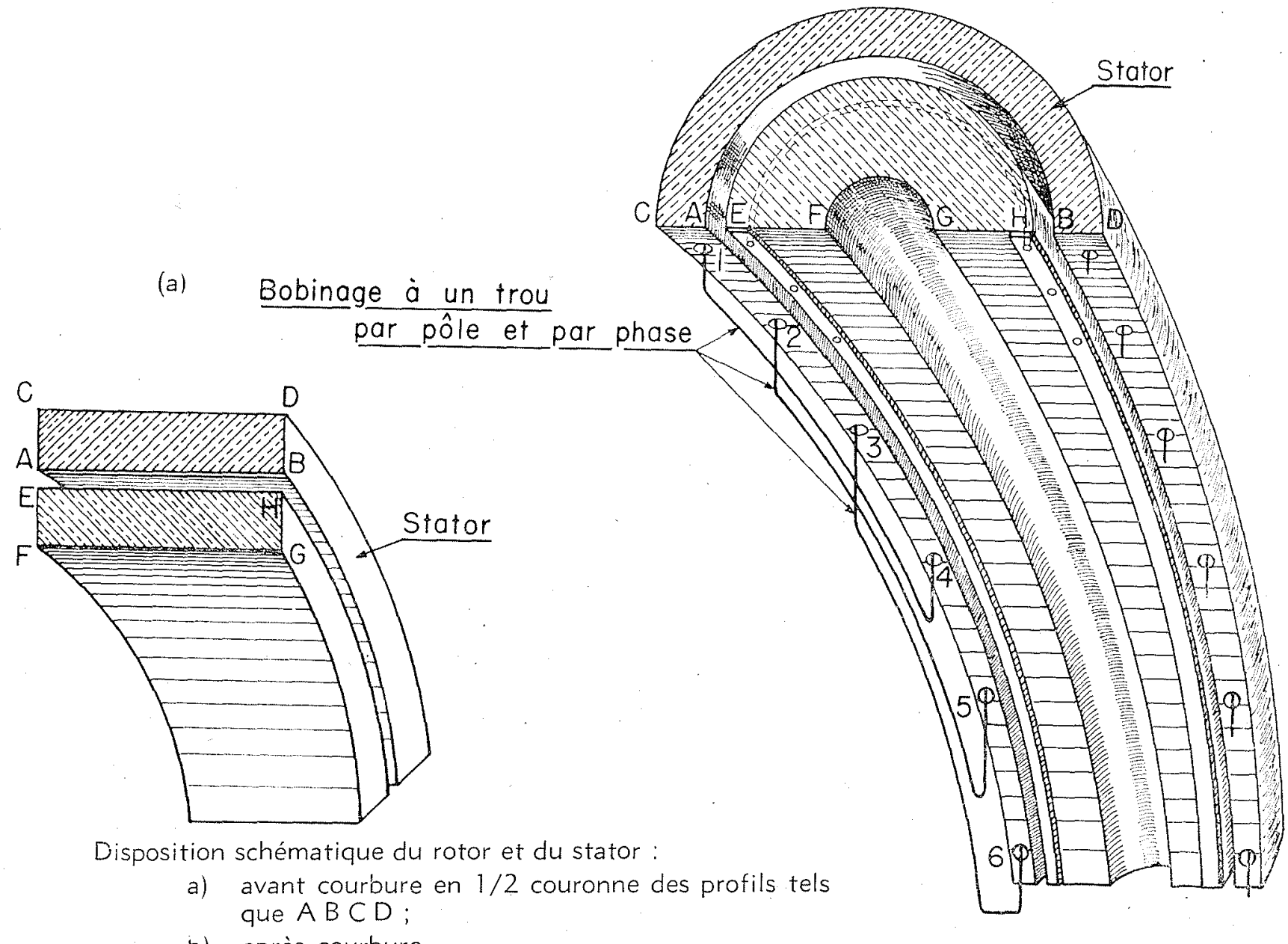

b) après courbure. 
a l'induit (1) comme dans la dynamo POIRSON ou dans un transformateur. Toutefois, dans la figure 9 qui montre les grandes lignes d'un électrogénérateur (ou moteur) type $B$, on a figuré pour plus de clarté un bobinage classique
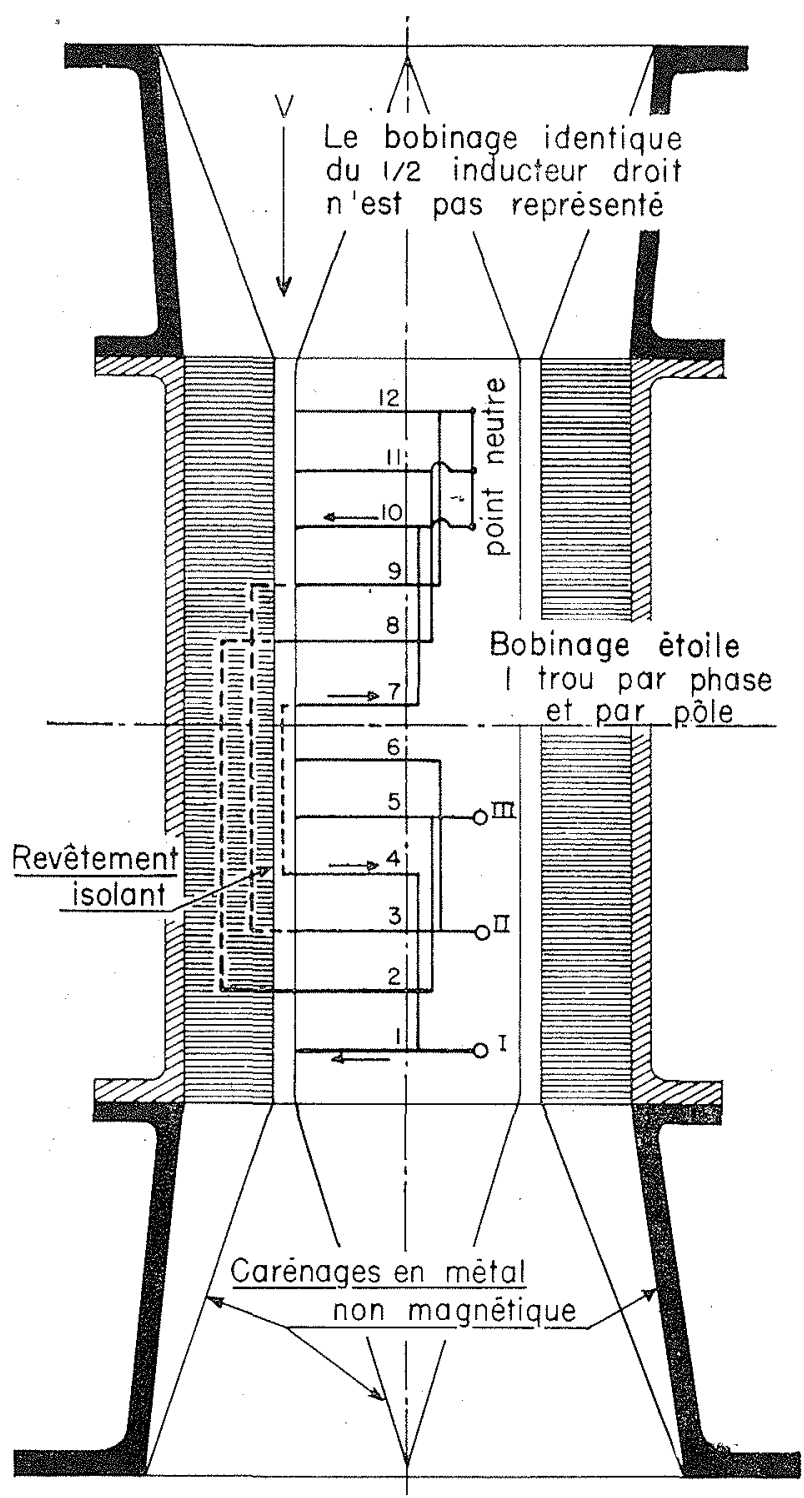

Fig. 9

Electrogénérateur bipolaire triphasé type «B *

a 1 trou par pôle et par phase sur la seule moitié supérieure de l'inducteur.

$C^{\prime}$ est le générateur type B qui paraît a priori le mieux adapté aux applications car il permet-

(1) Tout comme dans l'électrogénérateur type $A$, le bobinage inducteur peut être disposé soit sur le cylindre intérieur, soit sur la couronne d'acier qui l'entoure au mieux des facilités constructives. trait le transfert à un réseau HT d'une partie de l'énergie d'une veine fluide ou réciproquement sans contacts électriques entre le fluide et celuici. Toutefois il faut bien reconnaître qu'en pratique le transfert au fluide conducteur de l'énergie disponible au pied d'une conduite forcée ou à la sortie d'une chaudière pose des problèmes difficiles si l'on veut obtenir un bon rendement, par un appareillage robuste et peu coûteux.

\section{C. - L'ÉLECTROGÉNÉRATEUR \\ FLUIDOCINÉTIQUE COMME APPAREIL DE MESURE DE LA VITESSE D'UN FLUIDE}

\section{Généralités}

Les considérations exposées dans les paragraphes précédents montrent la possibilité $d^{\prime}$ induire dons des fluides en mouvement même mauvais conducteurs tels que les eaux naturelles des f.e.m. notables. Ces f.e.m. sont directement proportionnelles à la vitesse movenne du fluide si l'on maintient constant le champ magnétique et le champ des vitesses de l'écoulement (1). Elles peuvent être mesurées avec une grande précision par les nombreux microvoltmètres à lampes électroniques mis au point ces dernières années; ces mesures étant faites sans passage appréciable de courant, il est à prévoir - et l'expérience confirme - que la f.e.m. mesurée est indépendante de la résistivité du liquide en mouvement. II est donc tentant d'établir un système de mesure électrique des débits qui comporterait un électrogénérateur dûment simplifié relié à un microvoltmètre dont les indications donneront la vitesse du fluide qui le traverse grâce à une courbe d'étalonnage déterminée comme il est d'usage pour les moulinets hydrométriques par exemple. On peut mettre à l'actif de ce genre d'appareil les avantages suivants:

a) Possibilité de mesurer suivant la disposition donnée à l'électrogénérateur, soit des vitesses quasi-ponctuelles, soit des vitesses moyennes dans une surface relativement étendue.

b) Absence d'inertie quil permettrait d'enregistrer à l'oscillographe cathodique par exemple,

\footnotetext{
(i) Nous verrons plus loin que, dans certains cas tout au moins, la f. e. m. ne dépend pas de la distribution des vitesses.
} 
des vitesses à pulsations rapides (fluctuations turbulentes). L'appareil enregistreur ou à lecture directe pourra facilement être gradué directement en vitesses (courbé d'étalonnage généralement linéaire).

c) Possibilité dans certains cas (écoulement dans un tube de faible diamètre tel qu'une artère ou une veine) d'effectuer la mesure sans introduire aucune perturbation si petite soit-elle dans l'écoulement.

d) L'appareil résiste aux produits chimiques corrosifs aux suspensions grossières, etc...

e) La sensibilité de l'appareil peut être très grande; KOLIN a mesuré des débits de $1,6 \mathrm{~mm}^{3}$ s. dans un tube de $1 \mathrm{~mm}$. de diamètre.

Par contre, on peut prévoir à priori les inconvénients suivants:

1) La «Chaîne métrologique » reliant le «détecteur» à l'appareil de lecture est relativement longue. Les défauts de sensibilité et de fidélité du système de contrôle de la constance du champ magnétique, de l'amplificateur à lampes et du microvoltmètre s'ajouteront cux erreurs propres du détecteur.

2) Dans les mesures industrielles, on peut craindre l'influence de f.e.m. induites par des champs parasites et qui peuvent être du même ordre que la t.e.m. à mesurer. Si ces parasites sont d'intensité constante, on peut, il est vrai les compenser (voir débitmètre $\mathrm{KOLIN)}$; on réduira l'influence de certains d'entre eux par un blindage soigné des appareils.

3) L'appareillage, très acceptable au laboratoire, sera peut-être un peu délicat pour des mesures en usine, mais cet inconvénient va en diminuant au fur et à mesure des progrès de l'électronique industrielle.

La mesure électrique des débits constitue, à ma connaissance, la seule application de l'action des champs magnétiques sur les fluides en mouvement qui a été signalée par la presse technique. Je résumerai tout d'abord l'essentiel des publications de A. KOLIN et de B. THURLEMANN sur ce sujet. Ces auteurs ont étudié des appareils avec le point de vue et pour l'usage des physiciens et des biologistes. A la lumière de l'étude des électrogénérateurs esquissée dans la présente note, j'essaierai ensuite de rechercher le schéma de systèmes mieux adaptés aux mesures industrielles.
II

\section{Travaux de A. KOLIN \\ (Columbia University, New-York)}

Les premières publications de A. KOLIN (1) datent de 1936. Un article paru dans «The Rewiew of Scientific instruments» de mai 1945 (11) paraît donner l'état actuel de ses recherches sur les débitmètres électriques. II présente le débitmètre de haute sensibilité à courant alternatif dont la figure 10 montre le schéma.

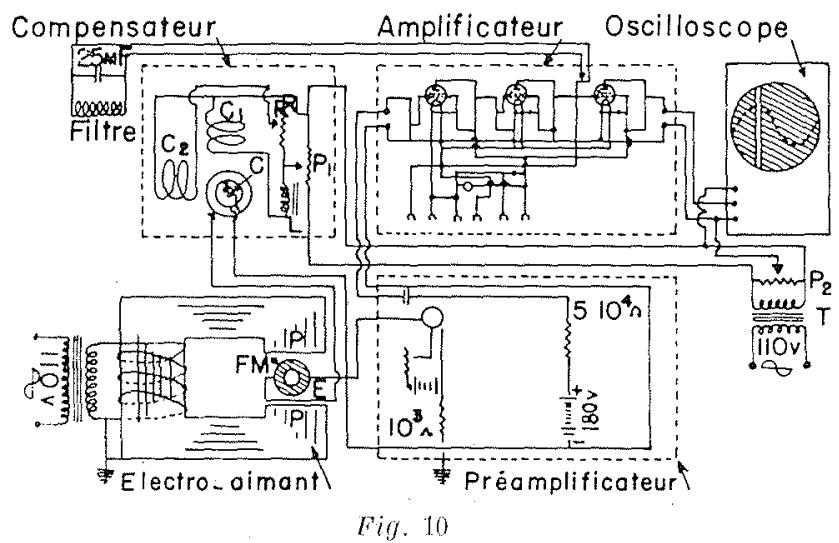

Le tube FM dans lequel passe le débit à mesurer est placé dans l'entrefer d'un électroaimant excité en courant alternatif. Deux électrodes $E$ en graphite ou en platine platiné sont disposées suivant un diamètre perpendiculaire à l'axe du tube et à la direction des lignes de force. Si le champ magnétique était constant au lieu d'être alternatif, l'auteur démontre par une méthode très générale là partir des équations de Maxwell et en utilisant le calcul vectoriel) que la différence de pośentiel mesurée entre les électrodes $\mathrm{E}$ est indépendante du profil des vitesses et de la conductibilité du fluide; en particulier cette différence de potentiel est la même que l'écoulement dans le tube soit laminaire ou turbulent, contrairement à ce que I'on déduirait d'un calcul élémentaire ne faisant intervenir que les f.e.m. :

$$
\begin{aligned}
d E= & \frac{\partial}{c} H V d r \quad \operatorname{avec} V=f(r) \\
& \text { et } E=2 \frac{\mu}{c} H \quad \int_{0}^{k} f(r) d r
\end{aligned}
$$

y. étant la perméabilité du fluide.

C la vitesse de la lumière.

$R$ le rayon du tube.

$r$ distance d'une particule élémentaire au centre du tube. 
Cette singulière propriété se maintient encore en courant alternatif, tout au moins en ce qui concerne la valeur maximum de la f.e.m. instan. tanée laquelle est réalisée comme l'on sait aux instants où $\frac{d H}{d t}=0$.

En définitive, quelle que soit la répartition des vitesses, la différence de potentiel $U$ entre les électrodes $E$ est égale ò la f.e.m. qui serait induite dans un conducteur de longueur $2 \mathrm{R}$ se déplaçant avec la vitesse moyenne $V_{m} d u$ fluide perpendiculairement à la direction des lignes de force du champ $\mathrm{H}$; on a donc:

$$
U=2 \frac{P}{\mathrm{c}} H V_{\mathrm{m}} \mathrm{R}
$$

soit en volts et pour $y=1$ toutes les autres grandeurs étant exprimés ên unités C.G.S. :

$$
\mathrm{U}=2 \mathrm{HRV}_{\mathrm{m}} \times 10-8
$$

Voici quelques résultats d'expériences de vérification effectuées avec du mercure; les différences de potentiel étaient mesurées par un galvanomètre:

\begin{tabular}{|c|c|c|c|c||}
\hline $\begin{array}{c}\mathrm{H} \\
\text { œrsteds }\end{array}$ & $\begin{array}{c}d \\
\mathrm{~cm} .\end{array}$ & $\begin{array}{c}v \\
\mathrm{~cm} . / \mathrm{sec} .\end{array}$ & $\begin{array}{c}\text { E calculé } \\
\text { microvolts }\end{array}$ & $\begin{array}{c}\text { E mesuré } \\
\text { microvolts }\end{array}$ \\
\cline { 1 - 3 } 1,350 & 0,150 & 231,0 & 467,0 & 470,0 \\
1,350 & 0,246 & 21,5 & 71,5 & 71,2 \\
1,350 & 0,395 & 9,97 & 53,2 & 53,4 \\
\hline
\end{tabular}

L'emploi d'un champ alternatif aux lieu et place du champ continu utilisé dans les premières expériences est dicté par le souci d'éviter l'emploi peu commode «d'électrodes non polarisables» pour capter la f.e.m. induite.

II présente, par contre, l'inconvénient d'introduire dans le liquide une f.e.m. «statique» de même nature que celle qui prend naissance dans le secondaire d'un transformateur industriel. Cette f.e.m. indésirable est annulée par un compensateur $C$ (analogues aux compensateurs industriels constitués par un moteur asynchrone calé) dont on règle la tension et la phase du secondaire pour que lorsque le liquide est au repos dans le tube FM I'appareil indicateur ou enregistreur de la vitesse soit au zéro. Dans le schéma de la figure 10, cet appareil indicateur est un oscilloscope monté avec amplificateur à lampes.

La figure 1 i montre l'une des dispositions adoptées pour le tube détecteur FM. Avec cet appareil (1) une lecture de $1 / 2 \mathrm{~mm}$. à l'oscilloscope correspond à un débit de $5 \times$ $10^{-3} \mathrm{~cm}^{3} / \mathrm{s}$; i en augmentant l'amplification on a pu déceler le débit correspondant à une goutte en $10 \mathrm{~s}$

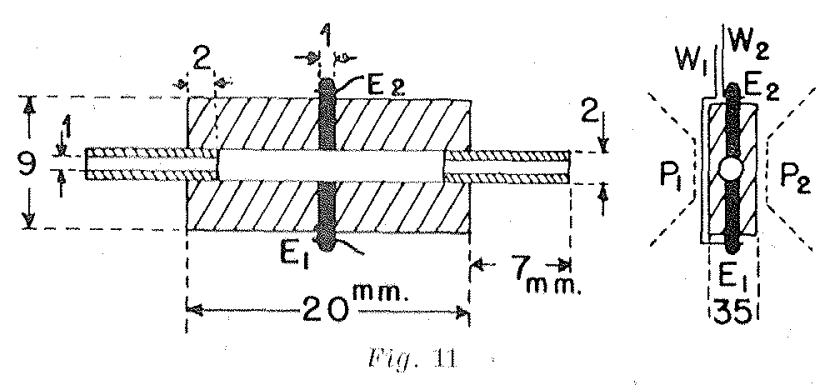

La figure 12 montre un autre dispositif réalisé à l'instigation du Professeur BAKMETEFF pour l'étude de la répartition des vitesses dans les grands canaux. Un électro-aimant miniature (noyaux de $1 \mathrm{~mm}$. de diamètre) crée un champ magnétique dans l'entrefer de $1 \mathrm{~mm}$. d'épaisseur de ses pièces polaires. Celles-ci portent 2 minces lames de mica verni au centre desquelles sont disposées les 2 électrodes $E_{1}$ et $E_{2}$. L'appareil mesure la vitesse moyenne dans l'étroit chenal

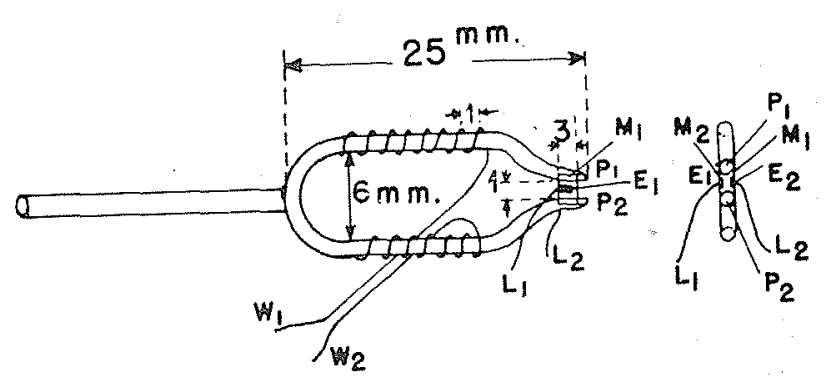

Fig. 12

de $1 \mathrm{~mm}^{2}$ de section compris entre les pièces polaires et les 2 lames de mica (vitesse quasiponctuelle); il fonctionne pour des vitesses de l'ordre de $25 \mathrm{~mm}$./s. et présente une inertie négligeable; il a été utilisé pour la mesure des fluctuations turbulentes de la vitesse de l'eau.

Remarque 1. - A. KOLIN fait observer que si l'on maintient la vitesse du fluide constante un appareil tel que celui de la figure 11 peut être employé pour la mesure de l'intensité d'un champ magnétique. Il y a là, peut-être, matière à quelques applications pratiques. J'ai pernsé

(1) Un tel appareil ne pourrait-il pas être utilisé à enregistrer les variations d'intensité de la pluie au cours des averses. 
notamment à la possibilité d'établir suivant ce principe un ampéremètre industriel pour les courants continus de très forte intensité. II serait constitué par un tube isolant placé dans le champ magnétique (éventuellement renforcé par un anneau en fer) produit par la barre transportant le courant à mesurer et dans lequel une petite pompe ferait circuler à vitesse constante un électrolyte quelconque. La mesure de la t.e.m. ainsi produite entre 2 points du fluide en mouvement serait proportionnelle au champ magnétique donc à l'intensité du courant. Les shunts utilisés actuellement pour lo mesure des fortes intensités sont encombrants, coûteux et ont tendance à "chauffer» (ils absorbent plusieurs kilowatts); le système proposé pourrait être plus précis, plus économique et éviterait la coupure des barres pour l'insertion du shunt.

Remarque II. - A. KOLIN attire encore l'attention sur le point suivant: Un diélectrique se déplaçant dans un champ magnétique sera polarisé. La polarisation induite sera donnée par l'expression (en notations vectorielles) :

$$
P=\left[k-\frac{1}{4 \pi c}\right][V, H]
$$

dans laquelle $k$ est la constante diélectrique et c la vitesse de la lumière. La polarisation induite est donc d'autant plus faible que la constante diélectrique se rapproche de 1 .

Dans un diélectrique se mouvant dans un champ magnétique à haute fréquence se trouveront donc induits des courants de polarisation qui pourront être utilisés pour la mesure du débit au moyen de dispositifs analogues à ceux que nous avons décrits pour les fluides conducteurs.

Il y a là, semble-t-il, un champ d'application intéressant à peu près inexploré.

\section{Travaux de B. THURLEMANN}

\section{(Thèse soutenue à I'Universiłé de Fribourg)}

Cet auteur a opéré uniquement en courant continu en utilisant des électrodes non polarisables au sulfate de zinc montées sur des tubes de faible diamètre. Pour la mesure de la différence de potentiel - en vue d'éviter l'emploi d'un voltmètre électrostatique qui serait évidemment l'instrument idéal dans ce cas THURLEMANN emploie une méthode d'opposi- tion "qutomatique ». A cet effet, un galvanomètre de zéro actionne un volet qui obture le faisceau de lumière tombant sur une cellule photoélectrique jusqu'à ce que le courant i convenablement amplifié, émis par celle-ci, crée aux bornes d'une résistance ad hoc une chute de tension "égale et opposée à la différence de potentiel à mesurer. Le courant i proportionnel à la différence de potentiel induite, mesuré par un appareil à cadre de type courant est exactement proportionnel à la vitesse moyenne du fluide dans le tube, quelle que soit la répartition des vitesses.

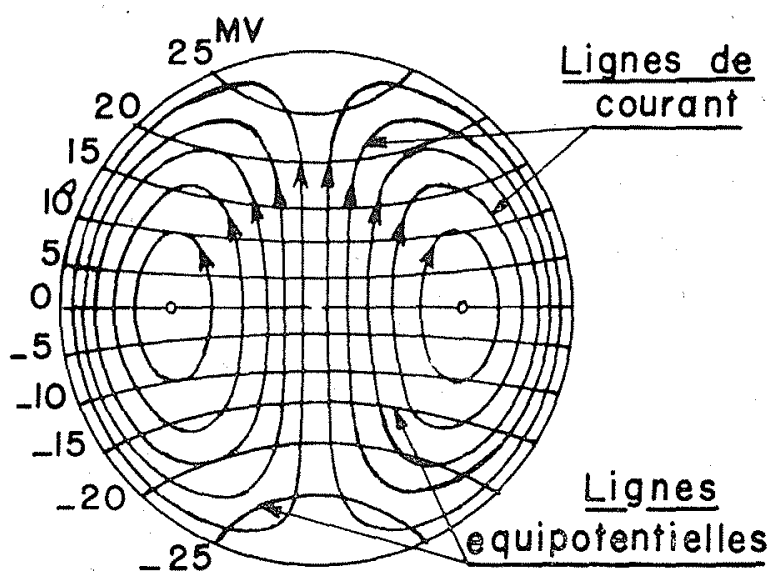

Fig. 13

La figure 13 donne d'après les calculs théoriques de l'auteur la répartition des lignes équipotentielles et des lignes de courant dans le tube parcouru par un écoulement supposé laminaire avec les valeurs numériques suivantes:

$$
\begin{aligned}
& H=10.000 \text { gauss } \\
& V=180 \mathrm{~cm} . / \mathrm{s} . \\
& \mathrm{R}=1,5 \mathrm{~cm} .
\end{aligned}
$$

Le liquide utilisé est une solution de $\mathrm{NaCl}$ à $8 \%$ dont la résistivité est de $10 \mathrm{ohms} / \mathrm{cm}$.

L'auteur a calculé le même réseau dans le cas d'un écoulement turbulent en admettant que la vitesse soit la même en chaque point de toute la section; les lignes équipotentielles seraient alors des cordes parallèles aux lignes de force et (pour une même f.e.m.) coupant la paroi du tube aux mêmes points que les lignes équipotentielles afférentes au régime laminaire; ce qui vérifie bien que les différences de potentiel entre 2 . points de la paroi ne dépendent pas de la répartition des vitesses.

La thèse donne des tableaux récapitulatifs des nombreux essais effectués par l'auteur pour 
vérifier cette dernière propriété ; il en ressort que la précision des mesures est assurée à 1,3\% près, les écarts maxima ne dépassant pas $2 \%$; mais l'appareillage utilisé ne comporte-t-il pas une trop longue «chaine métrologique» pour être vraiment précis?

\section{V!}

\section{Schémas d'appareils de mesure industriels.}

Les appareils décrits ci-dessus sont plutôt des appareils de laboratoire. J'ai cherché à établir le schémo de dispositifs susceptibles d'être utilisés pour des mesures industrielles.

\section{a) Mesure des débits.}

Le système utilisé dans les cellules débitmétriques de MM. A. KOLIN et THURLEMANN est inadmisible pour des tuyauteries dont le diamètre excède quelques centimètres. Pour des tuyauteries de quelques décimètres de diamètre, on pourrait utiliser un électrogénérateur du type A (voir figure 5) traversé par le liquide dont il s'agit de mesurer le débit. On allégerait l'appareil et on réduirait les pertes de charge en augmentant sensiblement l'épaisseur de l'entrefer sans toutefois dépasser une certaine limite de façon à ne pas trop s'éloigner d'un champ radial. L'induction pourrait être réduite vers 1.000 gauss sans que la mesure de la f.e.m. induite présente de grandes difficultés (voir page 642) ; pour les mêmes raisons et sous les mêmes réserves, on réduirait la longueur de l'inducteur qui serait alimenté en basse tension (12 ou 24 volts) et de préférence en courant alternatif afin d'éviter les sujétions d'emploi des électrodes non polarisables. L'appareil serait étalonné soit en usine, soit sur place par les méthodes usuelles.

\section{b) Mesure des vitesses.}

Pour des conduites de grand diamètre, le dispositif ci-dessus reste applicable, mais il serait probablement très onéreux. II sera préférable de le remplacer par un appareil mesurant des vitesses locales (à la façon des moulinets) à partir desquelles on calculera le débit par les méthodes habituelles.

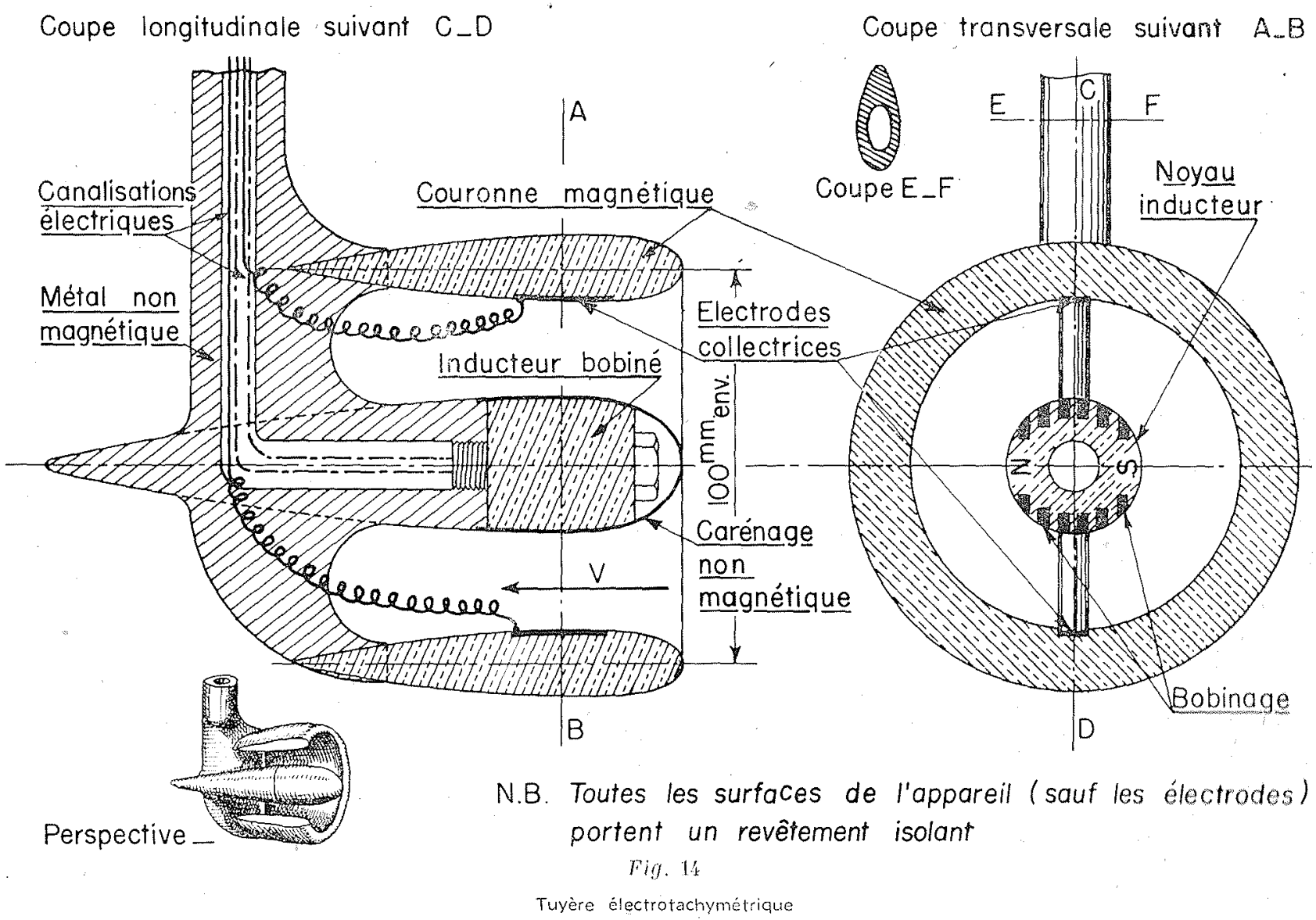


On pourrait, à cet effet, utiliser la cellule détectrice KOLIN-BAKHMETEFF représentée par la figure 12, mais ce dispositif paraît trop fragiie pour des mesures industrielles dans lesquelles il est d'ailleurs plus judicieux de relever, plutòt que des vitesses quasi-ponctuelles, des vitesses moyennes à l'intérieur d'une zone d'étendue proportionnée à l'aire totale à explorer. Dans cet esprit, j'ai esquissé l'étude de la «tuyère électrotachymétrique » représentée par la figure 14; cet appareil serait destiné à remplacer des moulinets hydrométriques dans toutes leurs applications pour autant que se trouveront palliés les inconvénients signalés page 646.

II dérive aussi de l'électrogénérateur type $A$. L'inducteur en métal magnétique est placé dans la partie antéro-médiane d'un carénage de révolution dont la directrice est un profil symétrique d'aile d'avion (profil Joukowski par exemple). Le champ magnétique radial de préférence alternatif se ferme par une culasse en métal magnétique entourant le noyau central et carénée suivant un profil analogue. Cette couroune porte les 2 électrodes collectrices. La figure 14 donne à titre indicatif une disposition constructive possible de l'ensemble. Le diamètre utile de la tuyère pourrait être de l'ardre du diamètre de I'hélice des moulinets $112 \mathrm{~cm}$. pour le modèle normal). L'expérience montrera si l'on peut supprimer la culasse magnétique qui entoure le noyau central et se borner à disposer les électrodes collectrices sous forme de 2 antennes symétriques reliées au noyau; la perturbation apportée au courant liquide par l'appareil serait oinsi moindre.

Peut-être pourrait-on remplacer l'électroaimant inducteur par un aimant permanerit, mais on se heurtera sans doute aux f.e.m. de polarisation consécutives à l'utilisation de courant continu dans le circuit induit.

Le tarage de la tuyère se ferait comme celui des moulinets en notant soigneusement la valeur du courant inducteur admise; en modifiant systématiquement cette valeur on pourrait ou besoin établir des appareils à plusieurs sensibilités.

L'un des avantages de ce type d'instrument serait de donner la vitesse $V$ (et dans une conduite forcée le débit $Q$ (si comme c'est généralement le cas, le profil des «vitesses relatives » he varie pas avec celui-ci) sous forme d'un courant électrique dont l'intensité est proportionnelle à $V$ :

$$
1=k V=k^{\prime} Q \text {; }
$$

cela permet facilement d'établir des instruments indicateurs ou enregistreurs gradués directement en $V$ ou en $Q$ ou de faire entrer systématiquement ces valeurs dans des appareils plus complexes. Ainsi si I'on envoie le courant I dans un compteur de quantité d'électricité genre $O^{\prime} K$, cet appareil indiquera directement l'intégrale:

$$
\int_{t_{1}}^{t_{Q}} Q d t
$$

c'est-à-dire le volume de fluide qui a traversé la section de mesure entre les instants $t_{3}$ et $t_{2}$. Nous donnerons plus loin un autre exemple.

Il serait facile d'établir sur le même principe une tuyère symétrique qui sans modification de son orientation dans le fluide indiquerait le renversement de sens du courant; monté sur girouette avec télétransmission de l'azimuth de celle-ci (système SELSYN) l'appareil pourrait rendre de bons services pour l'étude des courants marins; la salure de l'eau de mer qui est très gênante pour le fonctionnement des moulinets usuels serait pour la tuyère un élément favorable.

\section{c) Mesure des pressions.}

La possibilité de mesurer avec précision des vitesses de liquide infimes annoncée par A. KOLIN m'a suggéré l'idée d'utiliser un électrogénérateur comme manomètre par l'artifice suivant. Soit, pour fixer les idées, à mesurer les variations de pression à l'entrée d'une turbine hydraulique. Branchons au droit de la section de mesure une canalisation hydraulique débouchant dans le bief de restitution de la turbine et dimensionnée pour qu'elle soit le siège d'un écoulement laminaire (pratiquement, if faudra que les pertes à la Borda soient négligeables par rapport à celles répondant à la loi de POISEUILLE). Le débit q qui traversera cette canalisation sera proportionnel à la chute nette $H$ utilisée par la turbine diminuée du $\frac{V^{2}}{2 g}$ à l'entrée: on peut d'ailleurs éliminer cette dernière correction en alimentant la canalisation par une prise dynamique de tube de PITOT convenablement disposée. On aura donc:

$$
4=\mathrm{aH} \quad \text { a étant une constante. }
$$

Mesurons par une cellule du genre de celle de la figure 11, ce débit $q$; nous recueillerons aux 
bornes de l'appareil un courant I' proportionnel à $\mathrm{H}$ :

$$
\mathrm{I}^{\prime}=\mathrm{KH}
$$

et nous aurons réalisé un véritable manomètre.

Il semble possible de donner au système un délai de réponse très réduit et d'enregistrer aussi facilement des variations de pression assez rapides.

Supposons que nous alimentions les 2 bobines d'un électrodynamomètre (wattmètre) respectivement par le courant $1=k^{\prime} Q$ proportionnel au débit $Q$ à l'entrée de la turbine et par le courant $\mathrm{I}^{\prime}=\mathrm{KH}$ proportionnel à la chute nette de celle-ci. L'appareil donnera une indication proportionnelle au produit scalaire des vecteurs I, I'; c'est-à-dire à la puissance hydraulique instantonée entrant dans la turbine (compte tenu d'un éventuel déphasage entre $Q$ et $H$ si l'écoulement est pulsatoire). En combinant l'appareil ainsi constitué avec un équipage de wattmètre mesurant la puissance fournie aux bornes de I'alternateur accouplé à la turbine, il doit être possible de réaliser un instrument donnant le rendement global instantané du groupe. Les amplificateurs à lampes permettront toujours de disposer de courants 1 et $I^{\prime}$ suffisants pour le fonctionnement de tels appareils, mais en raison de la longueur de leur "chaîne métrologique 》 ils ne pourront donner une précision acceptable que que s'ils sont placés en de «bonnes mains».

Quoiqu'il en soit, cet exemple montre l'extrême souplesse de ces appareils électriques; il est très facile d'enregistrer directement les dérivées premières et secondes de $\mathrm{V}$ et de $\mathrm{H}$ :

$$
\frac{d V}{d t} \frac{d^{2} V}{d t^{2}} \frac{d H}{d t} \frac{d^{2} H}{d t^{2}}
$$

qui jouent un rôle important dans certains problèmes (régulation). Les vitesses mesurées avec les débitmètres $A$. KOLIN sont extrêmement faibles et correspondent à des gradients de pression évanescents. Ces appareils peuvent donc dans certains cas être utilisés comme micromanomètres. Il ne paraît pas impossible de les adapter à l'enregistrement de la houle à partir des pressions mesurées en profondeur (15).

Remarque. - Nous avons supposé que la canalisation de mesure de la pression était établie pour donner un débit proportionnel à $H$; dans certains cas - pour diminuer le délai de réponse des manomètres par exemple - il pourra être plus avantageux de constituer celle-ci par un étranglement donnant une perte de charge nettement quadratique; on aura alors :

$$
\mathrm{H}=\mathrm{kq}^{2}
$$

relation qui relie $I^{\prime}$ à $H$ par la loi en

$$
I^{\prime}=k^{\prime} \sqrt{H}
$$

qui est souvent moins commode que la loi linéaire mais parfaitement utilisable surtout dans un appareil qui devra subir forcément un étalonnage préalable.

\section{CONCLUSIONS}

L'exposé qui précède semble montrer que l'étude de l'action du champ magnétique sur les fluides erı mouvement peut conduire à diverses applications notamment dans le domaine des mesures.

Notre but sera atteint si cet exposé incite quelques chercheurs français à s'intéresser à ce domaine encore inexploré dans notre pays, mais qui fait dès maintenant l'objet d'études suivies à l'étranger.

\section{INDEX BIBLIOGRAPHIQUE}

$1-$ A. KOLIN. Proc. Soc. Exp. Biol. Méd. 35, 53-57 (1936).

2. E. WETTERER. Zeits. f. Biol. 98, 26 (1937).

3 - E. WETTERER.

Zeits. f. Biol., 99, 158 (1938).

4 - A. KOLIN.

A variable phase transformer and its use as an A. C. interference eliminator.

Rev. of scient. Industr. New-York (Nov. 1941) vol. 12, 555

5 - A. KOLIN

An A. C. induction flow meter for measurement of blood flow in intact blood vessels.

Proc. of the Soc. for Exper. Biology and Med. 46, No 2, 235-239 (Février 1941)

$6-$ A. KOLIN.

The flow of blood in carotid artery of the dog under various circumstances as determined with the electromagnetic flowmeter. Amer. J. of Physiol. Baltimore, 32, 788-804, (1938).

7 - B. THURLEMANN.

Méthode électrique de mesure de débit des fluides. Helv. Physica Acta, No 5-6 (1941)

Analysé dans "Mesures» (Décembre 1943).

$8-$ A. KOLIN

U. S. Patent No 149,847 (1939).

9 - A. KOLIN.

Electromagnetic velocity. I. A method for the determination of fluid velocity distribution in space and time.

1. of Applied Physics. 15, No 2, 150-164 (Février 1944). 
10 - E. POIRSON.

Production et emploi de courants continus à grande intensité.

- Dynamos à induction unipolaire ou acyclique R. G. E. (12 novembre 1938)

$11-$ A. KOLIN.

An alternating Field induction Flow Meter of High Sensitivity. The Review of Scientific Instruments. Vol. 16, No 5 (Mai 1945).

12 - M. PLANK. Electromagnétisme (trad. par E. LABIN). Félix Alcan, éditeur. Paris 1919.
13 - E. POIRSON.

Note sur la dynamo unipolaire étudiée et réalisée à I'Office National de Recherches et Inventions. Recherches et Inventions. Paris, avril 1933

$14-$ H. D. EINHORN.

Electromagnetic induction in water. Transactions of the Royal Society of South Africa. 1940, 28, page 383.

15 - R. W. GUELKE, C. A. SCHOUTE-VANNECK.

The measurement of sea-water velocities by electromagnetic induction. J. Instit. Electr. Engrs. Février 1947. Vol. 94. P. II. No 37, page 71.

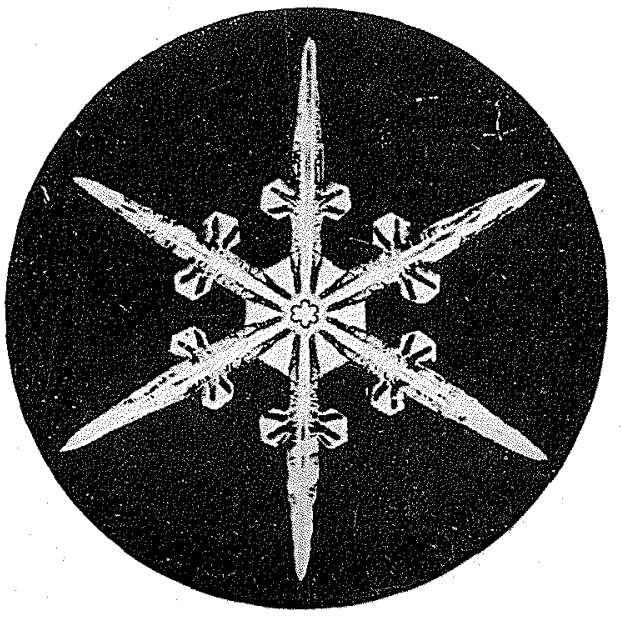

